\title{
Image Retrieval Algorithm based on Compressed Sensing and Dictionary Learning Methods
}

\author{
Xu Yang ${ }^{1, a}$, Ruiting Sun ${ }^{2, b}$ and Chenning $\mathrm{Yu}^{3, \mathrm{c}}$ \\ ${ }^{1}$ College of Computer Science, Sichuan University, Chengdu 610207, China \\ ${ }^{2}$ School of Electronics and Information Engineering, \\ Tianjin Polytechnic University, Tianjin 300387, China \\ ${ }^{3}$ Kuang Yaming Honors School, Nanjing University, Nanjing 210000, China \\ a835117276@qq.com, bsunrt95@163.com, crainorangelemon@gmail.com
}

Keywords: Image retrieval, semantic information, Nyquist sampling rate, weighted distance, precise image retrieving, cognitive theory, bi-cubic interpolation (BI).

\begin{abstract}
Image retrieval is based on the description of image content. The content of the image can be divided into two categories: visual content and information content. The visual content corresponds to the physical representation of the image, such as color, shape, texture. The information content corresponds to the semantic representation of the image, such as the theme, characters, and scenes. This paper discusses the image retrieval algorithm based on compressed sensing and dictionary learning methods. Compressed sensing, also known as compression sampling or compressed sensing, is a new sampling theory which acquires the correct signal a sampling speed far less than the Nyquest sampling rate. Compressed sensing technology randomly samples the signal through the development of signal sparse features, and then reconstructs the signal through nonlinear perfect signal reconstruction algorithms. In this paper, compressed sensing theory is applied to image retrieval in the process of feature extraction and matching. Combining with the optimized dictionary learning, a new retrieval model reconstruction algorithm is established. Experiments show that our algorithm can achieve high compression ratio through compression perception of linear measurement process. Using weighted distance method to calculate the similarity of measured value of the image features, the precise image retrieving is realized.
\end{abstract}

\section{Introduction}

High quality pictures are required for high accuracy estimations, and their spatial determination is the key marker in the appraisal of computerized picture quality; a picture with a higher spatial determination contains more data substance. The level of picture details is a conclusive criteria in accuracy and the scope of utilizations in supreme photogrammetry. [1]. However, constrained by factors such as the cost of the high-end equipment and the optical diffraction, certain troubles exist in straightforwardly procuring high determination pictures.

To fulfill the requirement of picture determination utilizing single edge photogrammetry of nearby geospatial information, super determination picture remaking was reviewed in this study [2]. There are two sorts of single casing picture, super determination reproduction techniques: interpolation and learning. Customary interpolation techniques accomplish high resolutions by utilizing an interpolation part work and incorporate strategies, for example, the closest neighbor, bilinear interpolation, bi-cubic interpolation (BI), and spline techniques. To further improve the quality, researchers later proposed edge situated interpolation techniques [3]. Visual impacts by layout fitting after picture edge discovery has been vastly enhanced. A wavelet based interpolation technique was proposed and a wavelet change was utilized to portray edge focuses, derive extraordinary point coefficients at better scales, and remake high determination pictures [4] [5]. An edge manage interpolation calculation was proposed which depended on the minimum squares technique, where the edge highlight of an added picture was kept up in light of the edge control highlight of the versatile covariance. A multi-surface fitting-based interpolation calculation has also 
been proposed that could expand the edge clarity of an inserted picture; that calculation had great heartiness against commotion [6].

The interpolation technique is straightforward and relevant to numerous circumstances; however, it needs earlier data, can only improve picture visual impacts, and regularly experiences issues in recuperating high recurrence data that is lost in low determination pictures, creating obscured reproduced pictures. On the contrary, learning-based super determination reproduction is accomplished by building up comparing connections amongst high and low determination pictures utilizing earlier data. The super determination strategy analyzes earlier data between high and low determination pictures with the assistance of Markov irregular field. This kind of technique accomplishes fantastic result on human facial and word preparing applications [7]. The meager representation utilized the scanty qualities of characteristic pictures and direct programing to fathom for the inadequate representation of the low determination pictures and after that combined the acquired representation coefficient with a high determination lexicon to make high determination picture tiles [8]. A picture interpolation calculation was anticipated that depended on a nonlocal auto relapse demonstration under the system of scanty representation. This technique utilized comparative picture hinders that were fundamental in common pictures to build a nonlocal auto relapse demonstrate; it then utilized self-similitude as a part of the picture structure as extra data to remake high determination pictures [9].

Commence of the learning strategy is to acquire earlier information of the high determination pictures. This method starts with existing pictures, creates relating low determination pictures by means of obscuring and down testing, and afterward utilizes a remaking calculation to rough the first pictures [10]. The remade pictures regularly don't exhibit generous changes in picture quality or determination. Realizing the constraints of customary remaking techniques, this study utilizes the neighborhood and spatial relationship between the introduced and unique pictures, naturally combines the interpolation and learning strategies, and proposes a compacted detecting, word reference based, super determination reproduction strategy.

The proposed strategy regards a current picture as a low determination picture, utilizes BI pictures as aides, and makes a joint lexicon for picture preparing in light of the scanty attributes of advanced pictures by requiring that the high-and low-determination pictures have similar meager representation in the comparing high-and low determination word reference. At that point, the related management between the high and low determination pictures is connected to the BI picture, and the improvement issue is explained utilizing compacted detecting hypothesis. The proposed strategy can successfully recover more definite high recurrence data while creating a super determination picture.

\section{Model of Compressed Sensing}

Preparing sample extraction and word reference learning has been previously discussed. The objective of the super determination remaking of a solitary edge picture is to reestablish a given low determination picture to a high determination picture of similar view.

The other key component in super determination recreation is to reproduce the low determination lexicon's scanty representation as for the low determination picture tiles. Compacted detecting is another hypothesis in the field of flag handling. The Dimensional flag which is spoken to as "Ds" is a component of aggregate flags that are available. This can be communicated by the superposition of an arrangement of orthonormal premise and it is show in condition 1.

$$
\mathrm{Ds}=\varphi \mathrm{R}
$$

In Eq.(1), "R" with various nonzero components are available. There may be once in a while nonzero components are more prominent than the other acquired focuses, then dimensional flag is otherwise called the sparse signal.

The testing procedure of compacted detecting is accomplished by utilizing a detecting lattice that is not related to the premise. The $\mathrm{n}$ dimensional network is observed to be equivalent to the 
dimensional flag which thusly is equivalent to the framework of detecting. This has been entitles as sparse dictionary. The accompanying condition 2 gives the scanty word reference.

$$
\mathrm{Dm}=\Phi \mathrm{Ds}=\varphi \mathrm{R}=\mathrm{SDic}
$$

Where Dm is dimensional framework, $\Phi \mathrm{Ds}$ is = dimensional flag, $\varphi \mathrm{R}$ is grid of detecting and SDic is scanty word reference.

Flag remaking is the centre of compacted detecting. For a meagre flag, the inadequate coefficient "R" can be conversely determined with a high probability from the X-Dimensional (X-D) perception esteem and the scantily imperative condition when the detecting framework fulfils the isometric condition. The first flag "S" can then be re-established with a high probability in light of the reversibility of the orthogonal change. Along these lines the flag recreation issue can be considered as a scan for the sparsest answer for the underdetermined condition.

\section{Projected Movement with Resolution Reconstruction}

The study utilizes the BI picture of a current picture as the high determination picture $\mathrm{HR}_{\text {image }}$. At that point, $\mathrm{HR}_{\text {image }}$ is prepared by similar obscuring and down examining as in the preparation stage, to get the relating low determination $\mathrm{LR}_{\text {image }}$. In view of the point by point attributes of the $\mathrm{BI}$ that stay unaltered in spatial area, the lexicon got in the preparation stage is straightforwardly connected to the more elevated amount BI picture. The super determination remaking issue can be depicted as the issue of illumination for the ideal estimation of the mind blowing determination picture $\mathrm{SR}_{\text {image }}$ By finishing the high and low determination lexicons, the super determination remaking procedure can be outlined. The interpolation administrator is utilized to play out a BI of low determination that creates the resultant, which has an indistinguishable determination from that of super determination picture. Similar strategy has been utilized for decaying the picture. The pictures are further deteriorated into tiles. Utilize the picture tile sparsity as earlier data.

\section{Experimental Outcome}

This study evaluates the inadequate representation coefficient of the low determination trademark obstruct with deference of the word reference through the ideal orthogonal coordinating interest calculation.

Source of the information is initially investigated. The premier undertaking in accomplishing super determination remaking is to build a high determination preparing test. In this concentrate, arbitrarily chosen common pictures are utilized as preparing tests. To confirm the adequacy of the super determination recreation model and calculation and examine the aftereffects of the super determination reproduction of thick backwoods pictures utilizing the proposed technique having a determination of $10 \mathrm{~cm}$, and another picture of determination $300 \mathrm{~cm}$, and a nearby up picture have a determination $5 \mathrm{~mm}$ that is being gathered by nonmetric computerized camera are utilized as a part of the super determination remaking test in this study. The outcomes are contrasted with those created by the BI technique. The transformation from RGB to YUV is performed at first as the whole pictures considered are shaded. The super determination remaking part utilizes proposed strategy; while the other two channels utilize BI technique lastly reconvert the hued picture to RGB space. To expand the speed of the trial, a test picture with a size of $255 \times 152$ pixels is utilized for this super determination explores.

The parameters are set in Matlab to get the best result. The preparation picture is utilized as a high determination picture, and the comparing low determination picture is gotten by down inspecting, where the down testing component 4 , and a one measurement channel is utilized to obscure the preparation picture in the event and vertical headings, individually. The picture is then amplified utilizing the BI technique, and the preparation test set is made out of 1000 picture tile combines that were extricated amid the test. 
The extent of a low determination picture tile is $5 \times 5 s$ pixels, and the cover is singular pixel. The quantity of cycles of the word reference match learning process utilizing the k-implies solitary esteem disintegration calculation is 25 , and the lexicon size is 1024 . In the testing stage, the BI strategy is utilized to amplify the picture three times, making the high determination test picture 'Ti'. At that point, the proposed strategy is utilized to accomplish a remaking that has a super determination that is three times bigger.

\section{Results and Analysis}

The pictures for the analysis considered frame thee thick woods. The principle center has been focused on reproduction exactness. To break down the effect of various ground include sorts on the picture, thick woods picture is utilized as source information for catching pictures of ordinary ground components. For example, some of the pictures that are used are the thick timberland with sun beams, woodland at frosty season, etc. The recreation results are appeared in Figure 1. For examination, the first picture is amplified utilizing the closest neighbor interpolation technique. To assess the nature of a shading picture, the human target impact is solid. As appeared in the subsequent pictures, the picture created by the customary BI strategy is obscured, and the reproduced picture needs clear points of interest at edges and exhibits a sporadic basic district with genuine misfortunes of surface. The remade picture delivered by the compacted detecting strategy is clearer; the privately amplified picture that is ordinarily bigger shows more point by point surfaces and an enhanced edge impact.

The quantitative record estimations of the reproduced pictures delivered by the BI technique and the proposed strategy are examined. Contrasted with the BI strategy, the packed detecting technique exhibits certain changes in both the data entropy and normal differentiation. The data entropy of the average components, for example, the thick backwoods with sun beams, timberland at icy season increments, and the picture differentiate likewise increments.

The data entropy of the picture reproduced by the proposed technique increments by 0.1 , and the normal picture differentiate increments by 0.6. To depict the level of upgrade of different ground highlight picture reproduction records, the expanded qualities are drawn into related bends in light of ground highlight sorts. Figure 2 indicates Curve in view of various elements of thick woodland with information entropy. Figure 3 demonstrates the Curve in light of various elements of thick backwoods with normal differentiation.

Concerning picture data entropy, the entropy step by step increments from working to vegetation, uncovered soil, and water body. The expansion in entropy is most set apart for the water body. As far as picture differentiation, the expansion in vegetation is the most predominant, while that of the water body is slightest pervasive. 


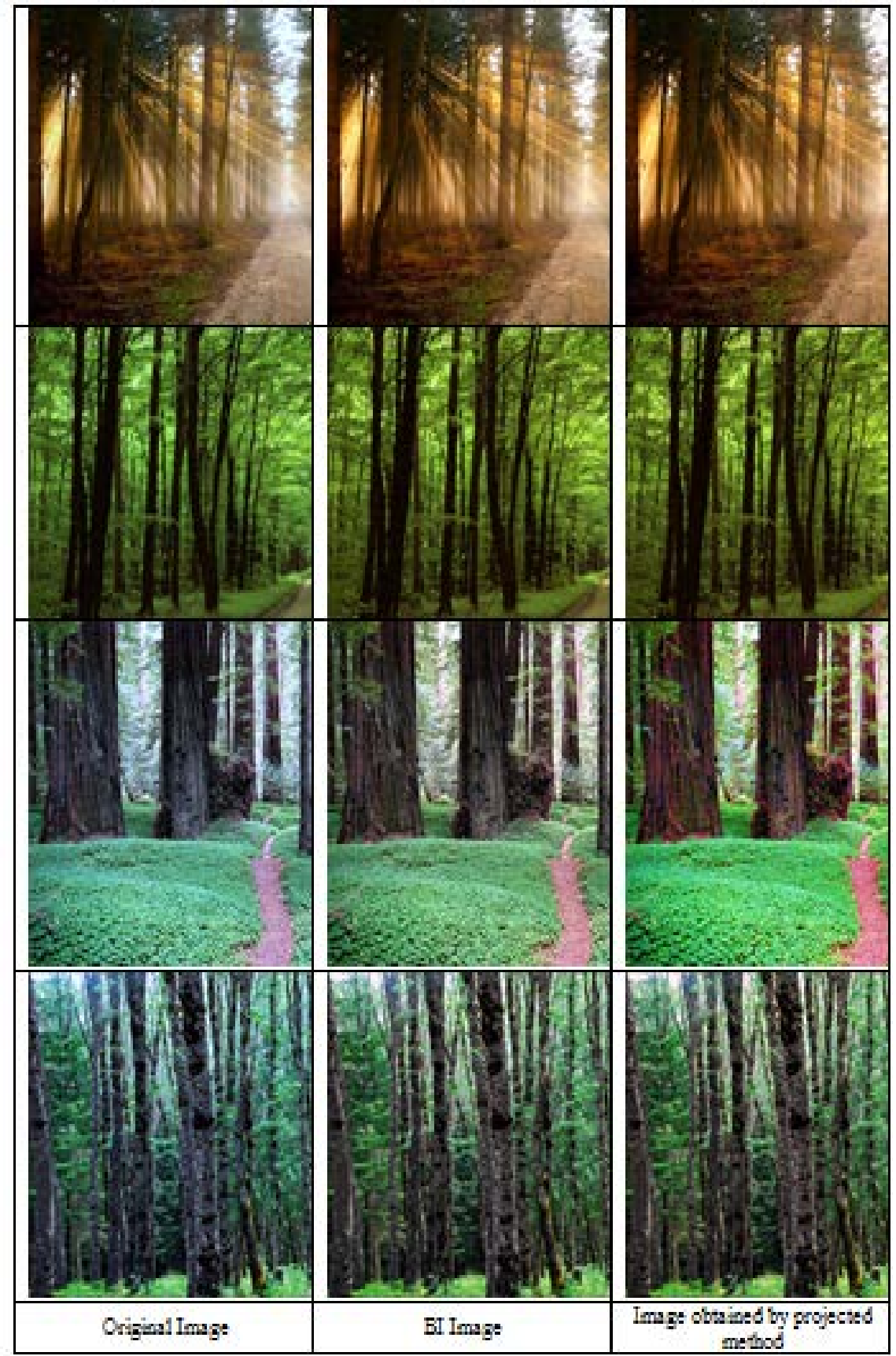

Figure 1. The original and the resultant images

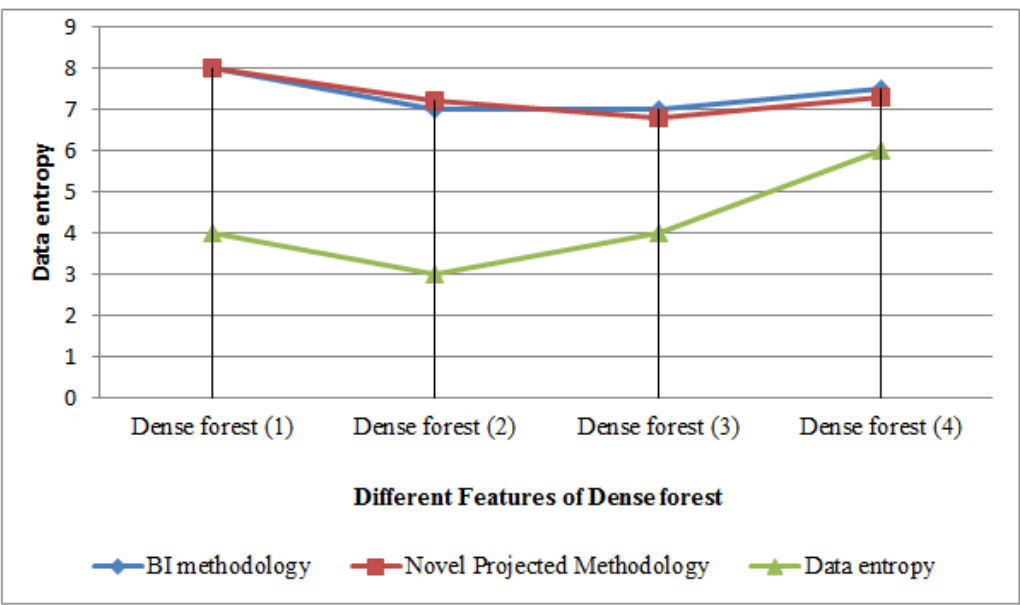

Figure 2. Curve based on different features of dense forest with data entropy 


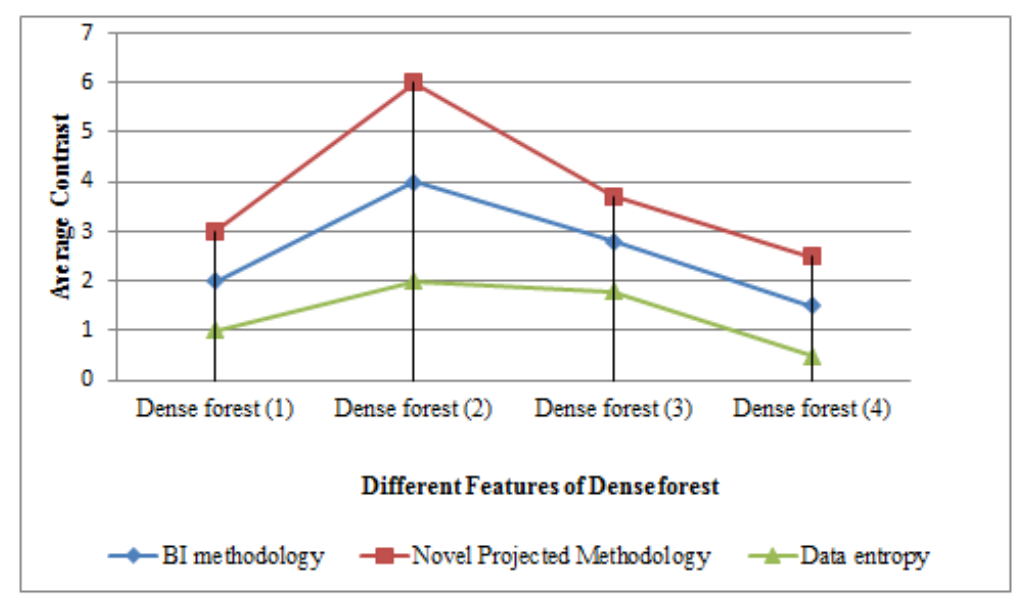

Figure 3. Curve based on different features of dense forest with average contrast

\section{Discussion}

Intensive study on the super determination reproduction of single casing pictures utilizing the meager representation and word reference learning strategies have been discussed. The versatile inadequate area and versatile regularization parameters are utilized to enhance recreation precision utilizing the enhanced word reference learning strategy, and normally creates great reproduction results. The studies utilized existing pictures as high determination pictures, and the super determination remaking procedure is just sparse reclamation of the down inspected pictures; the picture determination did not get crucial change. Consequently, this study utilized packed detecting as a hypothetical build alongside the comparability of the high and low determination picture attributes to prepare high and low determination picture lexicons utilizing the $\mathrm{K}$ implies solitary element disintegration technique. Utilizing a BI picture as earlier data, the proposed strategy demonstrated a super determination remaking of the current pictures, which generated a picture with a determination triple of the first picture. The exploratory aftereffects of this study demonstrated that the picture acquired by the proposed recreation strategy expanded both in visual impact and in quantitative file contrasted with the picture reproduced utilizing the BI technique. The surface structure and dim scale variety of various sorts of ground elements are thought about contrastingly thick woods pictures, which definitely instigate contrasts in reproduction exactness. Accordingly, to accomplish super determination remaking, this study utilized a less measured determination picture of city as a trial protest make a super determination reproduction of average ground includes in the thick backwoods picture. This concentrate then directed a precision examination with the conventional BI technique in view of the quantitative list investigation.

The test aftereffects of this study demonstrate that the building picture has the most enhanced recreation result because of the clarity and extravagance of the picture. Furthermore, the subsequent pictures all exhibit certain degrees of change, and the change in the picture data entropy exhibits an expanding pattern from building, vegetation, and uncovered soil to water body. Bring down unique picture entropy delivers more stamped upgrades. Alternately, the expansion in picture difference is stamped on the grounds that vegetation exhibits the wealthiest shading data and the most continuous variety in dim scale. The surface of the water self-perception is the most monotone, and hence, its expansion interestingly is the least.

Moreover, the augmentation in picture differentiation of various ground highlights keeps up a reliable association with the self-complexity of the pictures of these elements. Picture spatial determination is an imperative list for judging the measure of definite data in a picture. The immediate motivation behind super determination remaking is to expand picture determination; in any case, the reproduction procedure is based on the premise of existing pictures, and in this way, the determination of the current picture will unavoidably impact the recreation exactness. Subsequently, these studies played out a super determination remaking investigate three pictures of various resolutions. The consequences of this study show that as the first picture determination diminishes the 
remade pictures exhibit increments in the quantitative files. In this way, the proposed super determination reproduction strategy is especially reasonable for low determination pictures.

\section{Conclusion}

This study utilizes compacted detecting as a hypothetical system; naturally combines the interpolation strategy with the learning technique, guided by BI pictures; and creates a super determination remaking of a current picture at three times the scale. Contrasted with customary BI strategy, the proposed technique expands the picture data substance and clarity. The proposed strategy gives a compelling approach to enhance the application range and precision of flying and aviation thick woodland pictures.

\section{References}

[1] Chang, S. G., Cvetkovic, Z., \& Vetterli, M. (2006). Locally adaptive wavelet-based image interpolation. IEEE Transactions on Image Processing, 15(6), 1471-1485.

[2] Zhou, F., Yang, W., \& Liao, Q. (2012). Interpolation-based image super-resolution using multisurface fitting. IEEE Transactions on Image Processing, 21(7), 3312-3318.

[3] Wang, Haoxiang, and Jingbin Wang. "An effective image representation method using kernel classification." 2014 IEEE 26th International Conference on Tools with Artificial Intelligence. IEEE, 2014.

[4] Wang, Jingbin, et al. "Multiple kernel multivariate performance learning using cutting plane algorithm." Systems, Man, and Cybernetics (SMC), 2015 IEEE International Conference on. IEEE, 2015.

[5] Liu, C., Shum, H. Y., \& Freeman, W. T. (2007). Face hallucination: Theory and practice. International Journal of Computer Vision, 75(1), 115-134.

[6] Yang, Jianchao, John Wright, Thomas S. Huang, and Yi Ma. "Image super-resolution via sparse representation." IEEE transactions on image processing 19, no. 11 (2010): 2861-2873.

[7] Dong, W., Zhang, L., Lukac, R., \& Shi, G. (2013). Sparse representation based image interpolation with nonlocal autoregressive modeling. IEEE Transactions on Image Processing, 22(4), 1382-1394.

[8] Yang, J., Wright, J., Huang, T. S., \& Ma, Y. (2010). Image super-resolution via sparse representation. IEEE transactions on image processing, 19(11), 2861-2873.

[9] Lustig, M., Donoho, D. L., Santos, J. M., \& Pauly, J. M. (2008). Compressed sensing MRI. IEEE Signal Processing Magazine, 25(2), 72-82.

[10] Tsaig, Y., \& Donoho, D. L. (2006). Extensions of compressed sensing.Signal processing, 86(3), 549-571.

[11] Li, C. M., Deng, K. Z., Sun, J. Y., \& Wang, H. (2016). Compressed Sensing, Pseudodictionary-Based, Superresolution Reconstruction. Journal of Sensors, 2016.

[12] PU, J., \& ZHANG, J. P. (2010). Super-Resolution through Dictionary Learning and Sparse Representation [J]. Pattern Recognition and Artificial Intelligence, 3.

[13] Yang, J., Wright, J., Huang, T., \& Ma, Y. (2008, June). Image super-resolution as sparse representation of raw image patches. In Computer Vision and Pattern Recognition, 2008. CVPR 2008. IEEE Conference on (pp. 1-8). IEEE.

[14] Dong, W., Zhang, L., Shi, G., \& Wu, X. (2011). Image deblurring and super-resolution by adaptive sparse domain selection and adaptive regularization.IEEE Transactions on Image Processing, 20(7), 1838-1857. 
[15] Jiancheng, Z., \& Wenting, Z. (2015). A New Algorithm of Image Super-Resolution Reconstruction Based on MOD Dictionary-Learning, Journal of Graphics, vol. 36, no. 3, pp. 402406.

[16] SHI, J., \& WANG, X. H. (2013). Image Super-Resolution Reconstruction Based on Improved K-SVD Dictionary-Learning [J]. Acta Electronica Sinica,5, 026.

[17] Li, Chun-mei, Ka-zhong Deng, Jiu-yun Sun, and Hui Wang. "Compressed Sensing, Pseudodictionary-Based, Superresolution Reconstruction." Journal of Sensors 2016 (2016).

[18] Wei, S., Shen, Z., Zhang, S., \& Liu, S. (2013). Moon rover image super-resolution reconstruction algorithm, Geomatics and Information Science of Wuhan University, vol. 38, no. 4, pp. 436-439. 